\title{
Influence of sludge layer properties on the hydraulic behaviour of gravel-based vertical flow constructed wetlands for primary treatment of sewage.
}

Khomenko, O. ${ }^{1}$, Dotro, G. ${ }^{1}$, Jefferson, B. ${ }^{1}$, Coulon F. ${ }^{1}$, and Bajón Fernández, Y. ${ }^{1 *}$

${ }^{1}$ Cranfield University, School of Water, Energy and Environment, Cranfield, MK430AL, UK

"corresponding author: y.bajonfernandez@cranfield.ac.uk

\section{Abstract}

Sludge accumulation on the first stage of French design vertical flow constructed wetlands has been reported to improve treatment performance by favouring even sewage distribution on the beds' surface and increasing water retention time. However, due to its relatively low permeability, sludge layer can restrict the hydraulic capacity of the wetlands, requiring careful consideration of the feeding and resting strategy in order to avoid system ponding. This study aimed to elucidate the impact that sludge layer properties have on its permeability and investigate artificial modifications that could enhance it. A positive impact of increased organic matter content on sludge permeability was observed, with a 3-times permeability increase for a 15 percentage points higher volatile solids content. The opposite effect was observed for total solids, where an increase of 19 percentage points led to a drop of $1.6 \times 10^{-16} \mathrm{~m}^{2}$ on permeability. The impact of different surface modifications on drying kinetics and sludge layer properties was studied as a means to accelerate sludge layer mineralisation. Artificial modifications that modify surface tension of the sludge layer have been proved to achieve greater permeability and faster mineralisation of the sludge, with potential to achieve higher hydraulic acceptance and reduced 
operational costs (lower sludge accumulation) if implemented in full scale vertical flow constructed wetlands.

Keywords: mineralization, organic matter, preferential flow pathways, solids, sludge permeability, vertical flow constructed wetlands.

\section{Introduction}

Two stage vertical flow constructed wetlands (VFCWs) (commonly known as "French design") are subsurface flow constructed wetlands (CWs) used for the combined treatment of sewage and sludge onsite. They are typically comprised of three constructed wetlands (CWs) on the first stage and two on the second stage, with only one bed in each stage in operation at a given time. The first stage filter beds target the removal of total solids (TS) and partial removal of organic matter (OM) while polishing and nitrification occurs on the second stage (Molle et al., 2005). In conventional CWs, the wastewater solids content impacts operation and may cause beds clogging (Filho et al., 2018). On contrary, the solids accumulated by settling and filtration on the first stage of VFCWs result in the formation of a sludge layer, which increases water retention time and improves water distribution, having a positive impact on system efficiency. When properly designed and operated the sludge layer will accumulate at a rate of $2-3 \mathrm{~cm}$ per year (Dotro et al., 2017). Mineralization of the sludge layer deposited on the surface takes places during the resting periods, reducing sludge layer height and allowing long-term operation of the wetlands with desludging frequencies of $10-15$ years.

Sludge layer mineralization is also linked to hydraulic acceptance of the filters, and mineralised sludge layers with higher dry matter content have been reported to favour the hydraulic capacity of VFCWs, while very wet sludge layers with high OM content 
can have low permeability, which can in turn limit water percolation through the bed (Arias López, 2013; Molle et al., 2005). Hydraulic limitations of the sludge layer have previously been reported to occur in young filters, with improvement of hydraulic acceptance after 2-3 years of operation. Sustained periods of operation under hydraulic overloading and ponding occurrence during start-up period can compromise sludge layer mineralisation because of a lack of aerobic degradation (Arias et al., 2014; Molle, 2014a). This is believed to be linked to sludge layer development and evolution, which involves organic matter degradation and formation of voids and cracks which act as preferential flow pathways (PFP) and improve permeability.

The vast majority of existing literature investigating the hydraulic behaviour of CWs and soils discusses reduced percolation due to (1) pores blinding by smaller particles (Langergraber et al., 2003), (2) reduced effective porosity because of permanent attachment of biofilm to the filter medium (Langergraber et al., 2003), (3) reduced effective porosity because of reed roots development inside the medium pores (Langergraber et al., 2003), or (4) sludge deposition on the surface bed (Molle, 2014b). Available information is typically focused on the impact of clogging and reduced porosity on the VFCWs hydraulic acceptance, with limited information about the impact of the sludge layer properties itself (e.g. TS, OM) on the hydraulic behaviour of the system. Work on sludge drying reed beds (SDRBs) has shown the initial composition of the sludge in terms of OM, water content, fats and oils are critical to determine its suitability for treatment by wetland systems (Nielsen and Stefankis, 2019). Samples origin and composition can vary greatly between studies, making it difficult to evaluate the impact of individual properties for typical sludge layers. Similar to VFCWs, SDRBs require up to two years for the sludge layer to be sufficiently 
established before the design hydraulic loading can be applied without impairing the wetland system (Brix, 2017).

This study aimed to elucidate the influence of the sludge layer properties on its permeability and the rate of PFPs formation. Artificial means to accelerate PFP formation by sludge surface modification were investigated to evaluate the potential for reduced start-up periods and enable higher hydraulic acceptance of young filters. The impact of the sludge layer's TS, volatile solids (VS) and particle size distribution (PSD) on its permeability was investigated in constant head pressure filtration experiments. The rates of preferential flow pathways (PFP) formation and impact of sludge surface modifications were investigated in temperature controlled drying tests.

\section{Materials and Methods}

\subsection{Sample preparation}

Primary and secondary sludge were collected from a wastewater treatment plant located in the Midlands region serving a population equivalent of 2.5 million people. Primary sludge was used to replicate wetland sludge in permeability tests due to similarities in their composition. Similarly to wetland sludge, primary wastewater sludge accumulates as a result of primary precipitation of influent solids with typical VS content of $75 \%$ (Cleverson et al. 2007) comparing to $64-75 \%$ reported for young full-scale VFCWs (Gomez 2016).

The impact of VS and TS on sludge permeability was investigated using modified primary sludge to assess a range of both parameters. Aerobic degradation was employed to obtain a range of VS, for which samples where aerated by means of a diaphragm air pump (air flow $60 \mathrm{~L} / \mathrm{h}$ ) and two fine bubble diffusers for 5, 10, 15, and 20 
days. A range of TS content was achieved by centrifugation (Sorvall Legend RT, Kendro Laboratory Products, Asheville, US) during 60 minutes and further dilution of the samples. A concentrated sample was generated and supernatant from the centrifugation step was used for dilution to avoid chemical changes in the samples.

Primary sludge with a VS content of $75 \%$ was used in the drying tests. Secondary waste activated sludge ( $78.3 \%$ VS, $5 \%$ TS) distributed on the surface of the primary sludge was used as one of the artificial modifications for crack and PFP formation. Previous studies of rheological characteristics of the secondary and primary sludge and their blends showed differences in viscosity and stress yield (Baroutian et al. 2013; Markis et al. 2013) which were hypothesised to impact behaviour of the surface layer during drying.

\subsection{Sludge layer permeability}

The permeability of sludge samples was determined using a method based on Darcy's law for the flow of liquid through an already formed filter (sludge) cake (Li et al.2005). A constant pressure filtration rig with a tube diameter of $35 \mathrm{~mm}$ was used for the test (Advantec KST 47, Japan). The rig was connected to a compressed air source, which provided constant pressure of 0.4 bar in the tube (Guglielmi et al. 2010). The pressure value was recorded using a mechanical manometer (18-013-989, IMI Norgren, Birmingem, UK). A scale (Practum 3102-1S, Sartorius, Göttingen, Germany) placed under the constant pressure filtration rig allowed for the filtrate amount to be continuously recorded. The tube was loaded with a sludge layer of 5-7 mm height and filled with $0.01 \mathrm{M} \mathrm{KCL}$ solution $(1413 \mu \mathrm{S} / \mathrm{cm})$. A KCl solution as opposed to water was used to avoid any impact of the water ionic composition and conductivity on the sludge flocs properties. Each test was run in triplicates; with the sludge sample in the infiltration rig being replaced for each replicate. 
The sludge layer permeability was calculated using the equation (Li et al., 2005):

$$
k=\frac{\mu \cdot Q \cdot 1}{p \cdot A}
$$

where A is filtration area, $\left(\mathrm{m}^{2}\right) ; \mathrm{k}$ - permeability, $\left(\mathrm{m}^{2}\right) ; \mathrm{I}-$ cake thickness, $(\mathrm{m}) ; \mathrm{p}$ applied pressure, $(\mathrm{Pa}) ; \mathrm{Q}$ - total discharge over the test period, $\left(\mathrm{m}^{3} / \mathrm{sec}\right) ; \mu$ - viscosity, $(\mathrm{Pa} \cdot \mathrm{s})$.

\subsection{Preferential flow pathways formation}

In the current study formation of PFPs was investigated by performing temperature controlled convective drying experiments. Thickened primary sludge (TS content of $12-14 \%)$ with and without surface modifications was used. The initial TS content of the sludge samples was adjusted to a TS content close to that reported in literature for wetland sludge, which depending on the system age varies between $8 \%$ for a system operated for $<1$ year (Gomez 2016) to $26.4 \%$ for mature sites operated for $>10$ years (Molle et al. 2006). A TS value closer to young VFCWs was selected as the study aimed to understand mineralisation in the early system operation and investigate means to accelerate it. Several sludge layer modifications targeting modification of the sludge layer surface tension were applied (Table 1). Surface tension distribution was modified by forming initial cuts (PFP2 - PFP3) (Tao et al. 2005) on the sludge layer surface and by placing wooden cylinders of small diameter (PFP4) to mimic tension distribution in planted beds. PFP5 and PFP6 samples were modified by changing sludge rheology which defers for primary and secondary waste activated sludge (SAS) (Baroutian et al. 2013) and was hypothesised to impact surface tension hence changing sludge behaviour during drying. 
The sludge samples were placed into testing trays (Fig. 1a), with an initial sludge layer height of $4 \mathrm{~cm}$. For each condition tested three test trays were used for observation of changes in the sludge layer during drying, and one control tray was used for TS and VS content changes monitoring throughout the test.

Two dry warm air flows $\left(\mathrm{T} 60^{\circ} \mathrm{C}\right.$, relative humidity $\left.10 \%\right)$ from two identical sources were directed to the testing area avoiding direct contact of the air flow with the samples (Fig. 1a). The warm air flow was provided by two heat guns (AK06-GREEN, Aikou, China). The warm airflows were used to maintain conditions accelerating sludge layer drying and to prevent mould growth on the sample surface. Visual characterisation of the samples was performed during the test, with pictures of the samples surface taken at 30 minutes intervals and trays weight recorded for gravimetrical determination of sludge drying rate (Tao et al. 2005) until PFP is fully developed (when the crack reached the testing tray bottom). Moisture content in the sludge layer surface was measured in 3 zones of the testing tray: near wall (zone 1), at a distance of $1-2 \mathrm{~cm}$ from the wall (zone 2), and in the central part (zone 3) (Fig. 1b) by using a pin moisture meter (MO55, Extech Instruments, Massachusetts, US).

Sludge shrinkage with formation of a matte skin layer and formation of cracks were previously reported as typical changes in wastewater sludge during convective drying (Bennamoun et al. 2013) and were considered as critical points to be recorded in the current test. Two critical periods were determined for each drying test: initial period $\left(t_{1}\right)$, which comprises from the beginning of the test until appearance of the first crack, and crack development period $\left(t_{2}\right)$ - which is the period between occurrence of the first crack and full PFP development (when the crack reached the tray bottom). 


\subsection{Analytical methods}

Sludge samples were analysed for TS and VS content prior to permeability determination. TS and VS were determined in homogenised samples as per the standard methods (Standing Committee of Analysts (SCA), 2011) and expressed as a ratio of sludge weight before and after drying at $105^{\circ} \mathrm{C}$ when a steady mass was achieved. VS was expressed as \% of TS and measured by weight ratio of dried (for TS test) sludge samples before and after ignition at $550{ }^{\circ} \mathrm{C}$ until a steady mass was obtained. Particle size distribution (PSD) was characterised by laser diffraction spectroscopy. Tests were performed on a Malvern Mastersizer 3000 particle size analyser (Malvern Instruments Ltd, Malvern, UK) with a $30 \mathrm{rpm}$ pump speed and 150 rpm stirrer speed. Sludge concentration in tested solution was in a range between $0.1 \%$ and $0.6 \%$. The tests were run in triplicates for each sample. Fractal dimension was calculated according to reported soil fractal theory (Gao et al., 2014) based on the volume based size distribution data according to:

$$
\frac{V\left(r<R_{i}\right)}{V_{T}}=\left(\frac{R_{i}}{R_{\max }}\right)^{3-D}
$$

Where $r$ is the particle size, $R_{i}$ is the particle size of grade $i, R_{\max }$ is the maximum particle size, $V\left(r<R_{i}\right)$ is the volume of particles with a size less than $R_{i}, V_{T}$ is the total volume of the particles and $\mathrm{D}$ is the volume based fractal dimension.

\section{Results and discussion}

\subsection{Influence of sludge properties on its permeability}

Both TS and VS were found to influence sludge permeability. A decrease in sludge permeability with increasing TS content was observed across all sample sets (Fig. 2a). Permeability of non-aerated primary sludge (75\% VS content) decreased from $2.01 \times$ $10^{-16} \mathrm{~m}^{2}$ to $7.47 \times 10^{-17} \mathrm{~m}^{2}$ in samples with TS content of $9 \%$ and $24 \%$, respectively. 
The correlation remains true for all sample sets with the lowest permeability values observed for samples aerated for 20 days (60\% VS) with permeability decreasing from $7.75 \times 10^{-17} \mathrm{~m}^{2}$ to $4.56 \times 10^{-18} \mathrm{~m}^{2}$ with a TS range between 9 and $21 \%$.

Increased TS levels are linked to lower void fractions, which hinders water permeability. Besides, increased TS concentrations in sewage sludge have been reported to lead to floc disintegration, which in turn increases degree of dispersion and hinders sludge permeability because of an increased small particles fraction (Mikkelsen et al., 1999).

Unlike for TS, a decrease in VS content decreased the sludge permeability for the VS range tested of $60-75 \%$ (Fig. 2b). Permeability in sludge samples with $9 \%$ TS decreased from $2.01 \times 10^{-16} \mathrm{~m}^{2}$ to $6.14 \times 10^{-17} \mathrm{~m}^{2}$ when decreasing VS from $75 \%$ to $60 \%$, respectively, and from $1.25 \times 10^{-16} \mathrm{~m}^{2}$ to $2.06 \times 10^{-17} \mathrm{~m}^{2}$ in samples with TS content of $15 \%$ for the same VS range.

Particle characterisation revealed that aeration of the primary sludge aggregated the particles, shifting the particle size distribution to higher size fractions. To illustrate, the $\mathrm{d}_{90}$ increased from $42 \mu \mathrm{m}$ with unaerated primary to $385 \mu \mathrm{m}$ when aerated for 5 days whilst reducing the VS content by $3 \%$ (Table 2). Extending the period of aeration further reduced the VS content down to $60 \%$ and also showed expansion of the size distribution such that the $d_{90}$ reached a value of $512 \mu \mathrm{m}$ when the sludge was aerated for 20 days. Across the trial the volume based $d_{10}$ remained fairly stable or even decreased due to aeration and hence the samples retained a reasonable fraction of small particles even though substantial aggregation had occurred. Whilst aeration impacted the size of the aggregates, no significantly change in shape was observed. For instance, based on soil fractal theory utilising the volume based particle size data, 
the fractal dimension remained between 2.12 and 2.22 across the trial with the exception of the 15 day aeration period where the level was recorded to be much lower at 1.80 (Table 2). Comparison to reported data using the same method (Gao et al., 2014; Song et al., 2015) indicates the levels reported here are towards the low end of the scale indicating the structures are relatively open.

Overall, sludge mineralization (VS reduction) increased particle size $\left(d_{90}\right)$ without altering the structural openness of the flocs. Permeability decreased over this period suggesting that the broadening of the distribution of particle sizes imparted a significance influence over the ability to drain water through the sludge layer.

\subsection{Preferential flow pathways formation and sludge evolution during drying}

During the sludge drying experiments similarities in visual evolution were observed for the primary sludge without modifications and modified by forming initial cuts (PFP2 and PFP3), placing wooden cylinders (PFP4), dosing SAS (PFP5) and aerating (PFP6). There can be divided into three stages as follows: (1) sludge cake shrinkage and skin layer formation, (2) occurrence of the initial fissures and cracks, and (3) further development of cracks and sludge cake volume reduction. Formation of the skin layer occurs as water evaporates from the sample surface and moisture content in the surface layer decreases. A decrease in moisture content and formation of a matte skin layer occurred from the walls towards the tray centre. Moisture content decreased to $20-26 \%$ in zone 1 (Fig. 2b) during the first 30 minutes of the test and reached a value of $14-17 \%$ in zones 2 and 3 when the skin layer fully formed. While all samples seem to follow the 3 stages described above, PFP5 and PFP6 samples, modified by SAS application and aeration for 20 days, respectively, showed slightly different patterns. In these samples, formation of multiple cracks was observed in the 
shape of a cracked rind during the first 30 minutes of the test (Table 3 ), indicating a shortened first stage (cake shrinkage and skin formation) or its absence. However, not all cracks formed during the initial period evolved into fully formed PFP. Drying rates for PFP5 and PFP6 samples fluctuated during the remaining two stages, which may be explained by the formation of a large number of cracks throughout the test in the aerated primary sludge and in the samples modified by application of SAS on top of the primary sludge samples. The visual changes in sludge surface during these periods are shown in Table 2 and the drying kinetics changing over time are shown on the drying curves (Fig. 3).

Higher concentration of TS at the end of experiment, considered when a fully developed PFP was formed, was observed for all sludge samples where physical modifications were tested. The greater impact was observed for samples to which a layer of secondary waste activated was applied (PFP5), as a TS content of $39 \%$ was reached at the end of the test. TS concentrations in the final cake of $38 \%$ and $36 \%$ were recorded in samples modified with wooden cylinders of $1.5 \mathrm{~mm}$ diameter (PFP4) and aerated primary sludge (PFP6), respectively. For unmodified primary sludge (PFP1) a value of $27 \%$ TS was recorded at the end of the test. The impact of the artificial modifications on the kinetics of PFP formation was evaluated by comparing the PFP formation coefficient, expressed as a ratio of crack development period length $\left(t_{2}\right)$ to initial period length $\left(t_{1}\right)$. A lower PFP formation coefficient would indicate a positive impact of the sludge modification on evolution of the cracks to a fully developed PFP. The PFP formation coefficient was higher for samples PFP5 and PFP4 with a value of 0.8 and 1.2, respectively, compared to 0.3 and 0.5 for PFP3 and PFP2. PFP3 (initial III-cut) was hence the modification that led to a faster development of fully formed PFPs within those tested. 


\subsection{Implications of the findings for operation of full-scale VFCWs}

Previously reported literature for full-scale VFCWs indicate that sludge layers highly mineralised and with high dry matter content are characterised by greater infiltration rate (Arias et al., 2014; Pereira Gómez, 2016). The differences in hydraulic behaviour of the sludge observed during this study and those reported for full-scale systems is believed to be associated with the formation of PFPs, which are an inherent component of the system and naturally form as a result of biotic (Brix, 2017) or abiotic (Hsu et al., 2010) processes. These PFPs are characteristic of full scale systems and were absent in the sludge cakes tested in the permeability lab-scale experiments, which indicates a higher relative contribution of sludge layer structure (e.g. PFPs) than of its composition (e.g. TS, VS) to permeability.

The sludge layer properties observed to have a positive impact on sludge permeability (high VS and low TS) would restrict filter beds re-oxygenation and increase the sludge accumulation rate because of the greater volume of the sludge when its moisture is high. It is hence concluded that sludge layers characterised by a high degree of mineralisation (low VS, high TS) and with high abundance of PFPS are desired in full-scale systems, so that low sludge accumulation rates and high permeability can be simultaneously attained. Artificial means modifications of the sludge layer (surface tension or rheology changes, Table 1) that accelerate PFPs formation have the potential to avoid operational issues associated with low hydraulic acceptance of the first stage filters and increase operational time between desludging.

With a view to determine possible ways to stimulate formation of PFPs the impact of several artificial sludge layer modifications on sludge layer evolution during drying was investigated. The tests evidenced a positive impact of all the sludge surface 
modifications tested on the drying rates, which in full-scale systems could be implemented to improve TS evolution between feeding periods and hydraulic acceptance of the beds after the sludge layer is formed. Artificial formation of the initial cracks, addition of a SAS layer on the sludge surface and aeration of the sludge decreased PFP formation coefficient and moisture content in the sludge cake at the end of the experiment. Application of the activated sludge and aeration of the sludge caused positive changes in the sludge surface from the early stages of the experiments, with formation of multiple cracks that promote additional aeration of the sludge layer and generate a greater surface area, hence improving drying rates and mineralisation kinetics. No positive impact of the introduction of plant stem-sized pores on crack formation time was observed, although this modification had a positive effect on the TS evolution and formation of voids around the sticks in spite of the absence of wind-rock action typical of plant stems. This is expected to further contribute to preferential flow pathways in full-scale systems. Artificial sludge layer modifications could be valuable for both SDRBs and VFCWs, with the potential to allow a consistently higher hydraulic acceptance and reduce operational costs by enabling longer periods between desludging events associated with greater sludge mineralisation rates (Brix 2017).

A better understanding of the mechanisms by which sludge layer modifications change sludge structure and lead to formation of PFPs is still needed. In the case of SAS application on top of the sludge layer, which showed promising results, the relative contribution of rheology modifications and biotic processes to PFP formation requires further investigation, as well as the impacts of biological modification of the surface on system performance. 


\section{Conclusion}

A positive impact of increased VS and reduced TS content on permeability was observed in sludge samples with 60 to $75 \%$ VS and 9 to $25 \%$ TS. Sludge layer structure (PFPs) was concluded to have a higher relative contribution to permeability than its TS and VS composition. In order to simultaneously sustain long desludging frequencies and high permeabilities in full-scale systems, sludge layers characterised by a high degree of mineralisation (low VS and high TS) and high abundance of PFPs are desired.

Artificial modifications of the sludge layer accelerated PFP development and, if implemented in full-scale, could improve primary VFCWs hydraulic acceptance and shorten their maturation time. Higher TS contents were achieved for the artificially modified sludge samples, which could translate in prolonged operational periods without desludging (reduced maintenance costs) in full-scale VFCWs.

\section{Acknowledgements}

The authors would like to thank Severn Trent Plc. for their financial support.

\section{References}

Andreoli, Cleverson Vitorio Von Sperling, M., Fernandes, F., 2007. Sludge treatment and disposal. IWA Publishing, London.

Arias, L., Bertrand-Krajewski, J.L., Molle, P., 2014. Simplified hydraulic model of French vertical-flow constructed wetlands. Water Sci. Technol. L'Institut National des Sciences Appliquées de Lyon.

Arias López, J. luis, 2013. Vertical-flow constructed wetlands for the treatment of 
wastewater and stormwater from combined sewer systems. INSA de Lyon.

Baroutian, S., Eshtiaghi, N., Gapes, D.J., 2013. Rheology of a primary and secondary sewage sludge mixture: Dependency on temperature and solid concentration. Bioresour. Technol. 140, 227-233.

Bennamoun, L., Arlabosse, P., Léonard, A., 2013. Review on fundamental aspect of application of drying process to wastewater sludge. Renew. Sustain. Energy Rev. 28, 29-43.

Brix, H., 2017. Sludge dewatering and mineralization in sludge treatment reed beds. Water (Switzerland) 9.

Dotro, G., Langergraber, G., Molle, P., Nivala, J., Puigagut, J., Stein, O., von Sperling, M., 2017. Treatment wetlands. IWA Publishing, London.

Filho, F.J.C.M., Sobrinho, T.A., Steffen, J.L., Arias, C.A., Paulo, P.L., 2018. Hydraulic and hydrological aspects of an evapotranspiration-constructed wetland combined system for household greywater treatment. J. Environ. Sci. Heal. Part A 0, 1-8.

Gao, G.-L., Ding, G.-D., Wu, B., Zhang, Y.-Q., Qin, S.-G., Zhao, Y.-Y., Bao, Y.-F., Liu, Y.-D., Wan, L., Deng, J.-F., 2014. Fractal scaling of particle size distribution and relationships with topsoil properties affected by biological soil crusts. PLoS One 9, 1-10. doi:10.1371/journal.pone.0088559

Guglielmi, G., Chiarani, D., Saroj, D.P., Andreottola, G., 2010. Sludge filterability and dewaterability in a membrane bioreactor for municipal wastewater treatment. Desalin. 250, 1-9.

Hsu, J., Tao, T., Su, A., Mujumdar, A.S., Lee, D., 2010. Model for sludge cake drying 
accounting for developing cracks developing cracks 28 . doi:10.1080/07373937.2010.490775

Langergraber, G., Haberl, R., Laber, J., Pressl, A., 2003. Evaluation of substrate clogging processes in vertical flow constructed wetlands. Water Sci. Technol. $48,25-34$.

Li, W., Kiser, C., Richard, Q., Wenpinglifrmcocom, P.F.E., 2005. Paper presented at the American Filtration \& Separations Society 2005 International Topical Conferences \& Exposition, September 19-22, Ann Arbor, Michigan, in: Development of A Filter Cake Permeability Test Methodology. pp. 1-9.

Markis, F., Hii, K., Parthasarathy, R., Baudez, J.C., Slatter, P., Eshtiaghi, N., 2013. Rheological characterisation of blends of primary and secondary sludge, in: Conference: Chemeca 2013: Challenging Tomorrow. Brisbane, pp. 1-6.

Mikkelsen, L.H., Keiding, K., 1999. Equilibrium aspects of the effects of shear and solids content on aggregate deflocculation. Adv. Colloid Interface Sci. 80, 151182.

Molle, P., 2014a. French vertical flow constructed wetlands: A need of a better understanding of the role of the deposit layer. Water Sci. Technol. 69, 106-112.

Molle, P., 2014b. French vertical flow constructed wetlands: a need of a better understanding of the role of the deposit layer. Water Sci. Technol. 69, 106-112.

Molle, P., Liénard, A., Boutin, C., Merlin, G., Iwema, A., 2005. How to treat raw sewage with constructed wetlands: An overview of the French systems. Water Sci. Technol. 51, 11-21.

Molle, P., Liénard, A., Grasmick, A., Iwema, A., 2006. Effect of reeds and feeding 
operations on hydraulic behaviour of vertical flow constructed wetlands under hydraulic overloads. Water Res. 40, 606-612. doi:10.1016/j.watres.2005.11.026

Nielsen, S., Stefankis, A., 2019. Wetland Technology. Practical information on designing treatment wetlands. IWA Publishing, London.

Pereira Gómez, L., 2016. Vertical flow constructed wetlands for treating unscreened sewage in the UK. Cranfield University.

Song, Z., Zhang, C., Liu, G., Qu, D., Xue, S., 2015. Fractal feature of particle-size distribution in the Rhizospheres and bulk soils during natural recovery on the Loess Plateau, China. PLoS One 10, 1-15. doi:10.1371/journal.pone.0138057

Standing Committee of Analysts (SCA), 2011. Methods for the Examination of Waters and Associated Materials, Bluebook 236 British Standards. Environmental Policy and Law. doi:10.1016/S0378-777X(80)80046-1

Tao, T., Peng, X.F., Lee, D.J., 2005. Thermal drying of wastewater sludge: Change in drying area owing to volume shrinkage and crack development. Dry. Technol. 23, 669-682. doi:10.1081/DRT-200054164

Uggetti, E., Ferrer, I., Llorens, E., García, J., 2010. Sludge treatment wetlands: A review on the state of the art. Bioresour. Technol. 101, 2905-2912. doi:10.1016/j.biortech.2009.11.102

- Figure 1 Preferential flow pathways formation test layout. Red arrows show warm airflows.

- Figure 2 Relationship between sludge composition and permeability. 
- Figure 3 Drying curves of modified and non-mofied sludge samples : PFP1 (Thickened primary sludge with no modification) (a), PFP2 (Thickened primary sludge with initial V-cut) (b), PFP3 (Thickened primary sludge with initial IIIcut) (c), PFP4 (Thickened primary sludge modified with wooden cylinders of $1.5 \mathrm{~mm}$ diameter) (d), PFP5 (Thickened primary sludge with secondary waste activated sludge application) (e), PFP6 (Thickened aerated primary sludge with no surface modification) (f). The curves are plotted for single testing trays.

- Table 1 Sludge modifications used in preferential flow pathways formation test.

- Table 2 Characteristics of primary sludge with different aeration times.

- Table 3 Sludge layer evolution during drying. 
Table 1 - Sludge modifications used in preferential flow pathways formation test

\begin{tabular}{rll}
\hline Sample name & \multicolumn{1}{c}{ Sludge used } & \multicolumn{1}{c}{ Applied modification } \\
\hline PFP1 & Thickened primary sludge & No surface modification. Reference test. \\
\hline PFP2 & Thickened primary sludge & Initial V-cut \\
\hline PFP3 & Thickened primary sludge & Initial III-cut \\
\hline PFP4 & Thickened primary sludge & Wooden cylinders of 1.5 mm diameter \\
\hline PFP5 & Thickened primary sludge & Application of SAS layer of 2 mm on top \\
\hline PFP6 & Thickened aerated & No surface modification \\
& primary sludge & \\
& &
\end{tabular}


Table 2 Characteristics of primary sludge with different aeration times.

\begin{tabular}{ccccccccccc}
\hline $\begin{array}{l}\text { Aeration } \\
\text { period } \\
\text { length (d) }\end{array}$ & $\begin{array}{c}\text { VS } \\
(\%)\end{array}$ & $\begin{array}{c}\text { Relative } \\
\text { span }\end{array}$ & \multicolumn{4}{c}{$\begin{array}{c}\text { Particle size } \\
\text { distribution }(\mu \mathrm{m})\end{array}$} & $\begin{array}{c}\text { Relative } \\
\text { standard } \\
\text { deviation }(\%)\end{array}$ & $\mathrm{k},\left(\mathrm{m}^{2}\right)$ & $\begin{array}{c}\text { Fractal } \\
\text { dimension, D }\end{array}$ \\
\hline 0 & 75 & 0.9 & 17 & 28 & 42 & 1.1 & 1.2 & 2.8 & $1.34 \times 10^{-16}$ & 2.22 \\
\hline 5 & 72 & 4.5 & 17 & 82 & 385 & 2.7 & 0.9 & 0.2 & $1.28 \times 10^{-16}$ & 2.12 \\
\hline 10 & 70 & 4.3 & 21 & 99 & 447 & 2.6 & 1.1 & 1.4 & $5.82 \times 10^{-17}$ & 2.14 \\
\hline 15 & 67 & 4.8 & 19 & 89 & 448 & 0.4 & 0.5 & 0.7 & $3.43 \times 10^{-17}$ & 1.80 \\
\hline 20 & 60 & 7.8 & 13 & 64 & 512 & 3.1 & 0.7 & 1.1 & $1.55 \times 10^{-17}$ & 2.17 \\
\hline
\end{tabular}


Table 3 Sludge layer evolution during drying.

\begin{tabular}{|c|c|c|c|c|c|}
\hline Sample & . & $\begin{array}{c}\text { TS (\%) at the end } \\
\text { of the crack } \\
\text { development } \\
\text { period }\end{array}$ & $\begin{array}{c}\text { Beginning of the initial } \\
\text { period }\end{array}$ & $\begin{array}{l}\text { Beginning of the crack } \\
\text { development period }\end{array}$ & $\begin{array}{c}\text { End of the crack } \\
\text { development period }\end{array}$ \\
\hline PFP1 & 1 & 26.5 & & & \\
\hline PFP2 & 0.5 & 32 & & & \\
\hline PFP3 & 0.3 & 31.5 & & & \\
\hline PFP4 & 1.2 & 38 & & & \\
\hline PFP5 & 0.8 & 39 & & & \\
\hline PFP6 & 0.6 & 32 & & & \\
\hline
\end{tabular}


Highlights:

- Sludge layer restricts hydraulic capacity on vertical flow constructed wetlands

- Increased volatile solids and reduced total solids positively impact permeability

- Preferential flow pathways have higher contribution to permeability than composition

- Sludge layer modifications can improve VFCWs hydraulic acceptance

- Sludge layer modifications can prolong operational periods without desludging 
(a)

(b)

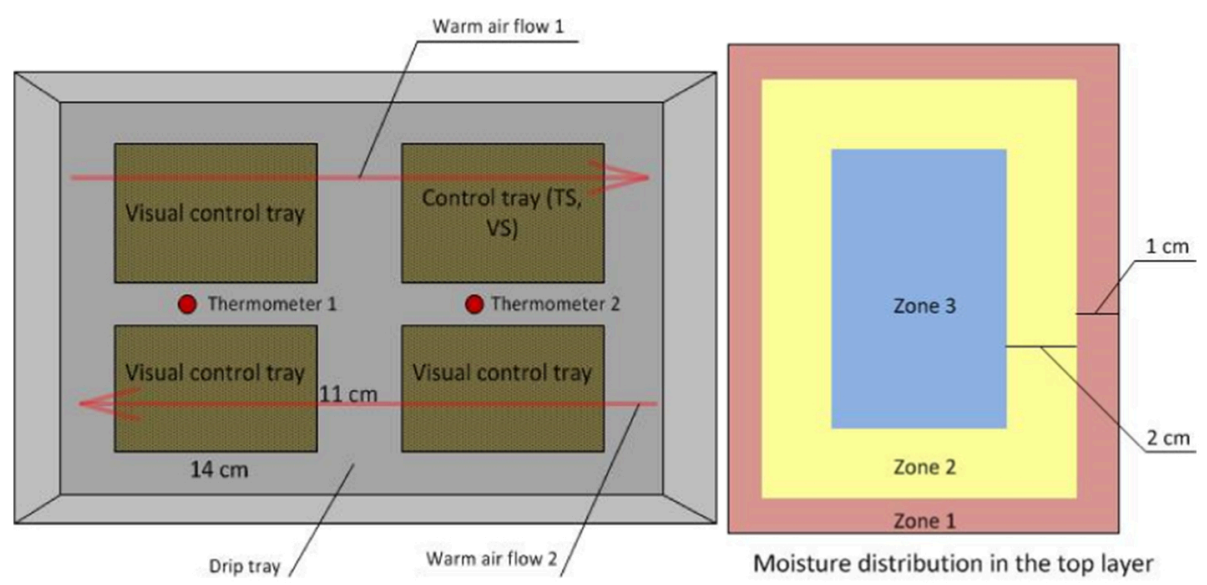

Figure 1 


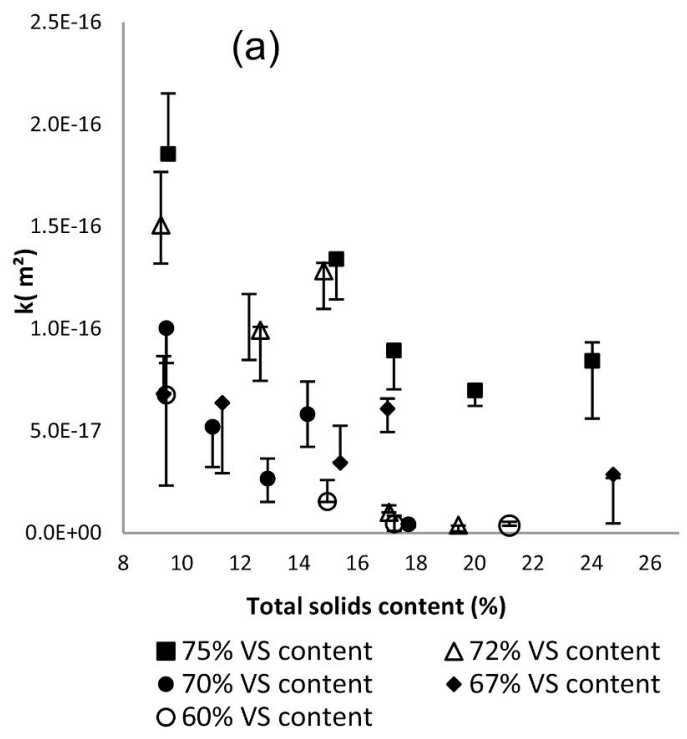

(b)

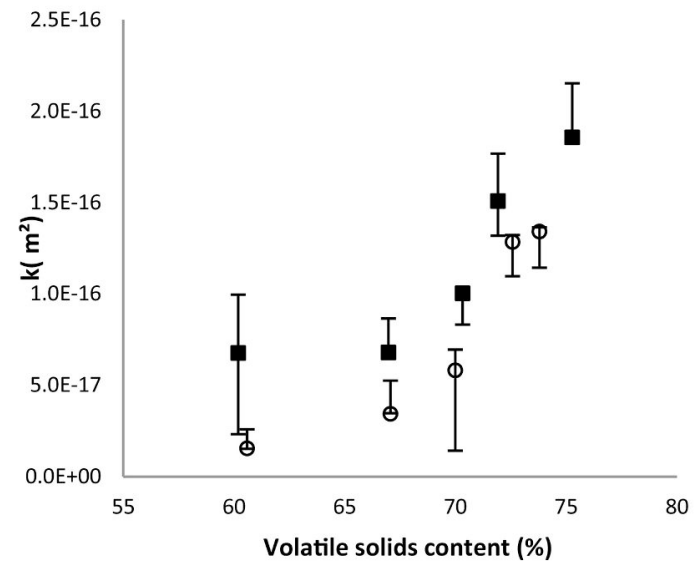

- 9\% TS content o 15\% TS content

Figure 2 
(a)

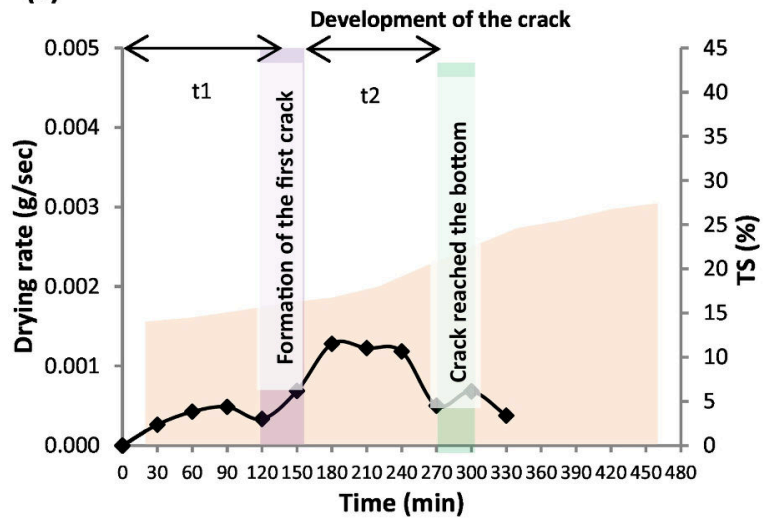

TS \% —Drying rate, $\mathrm{g} / \mathrm{sec}$

(c)

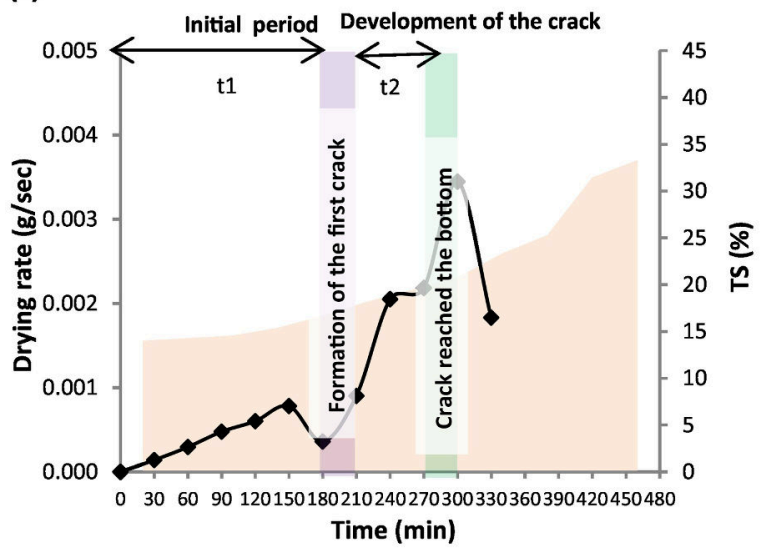

TS (\%) —-Drying rate, $g / s e c$

(e)

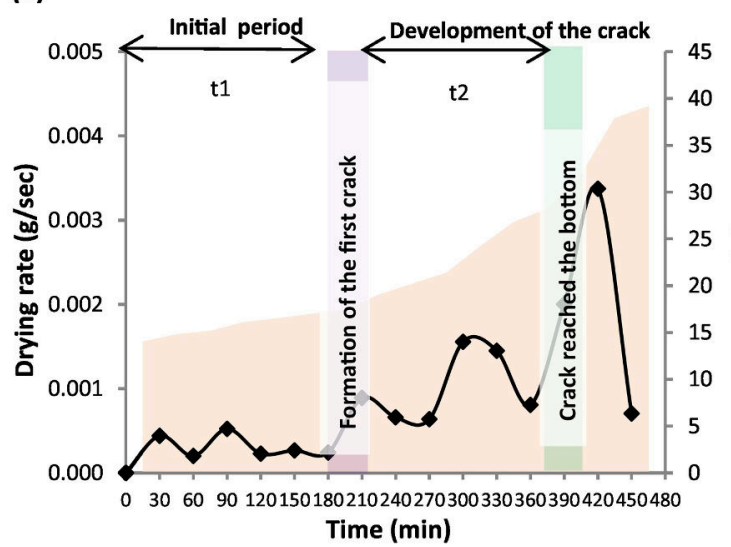

TS (\%) ——Drying rate, g/sec

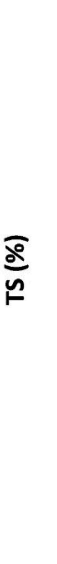

(b)

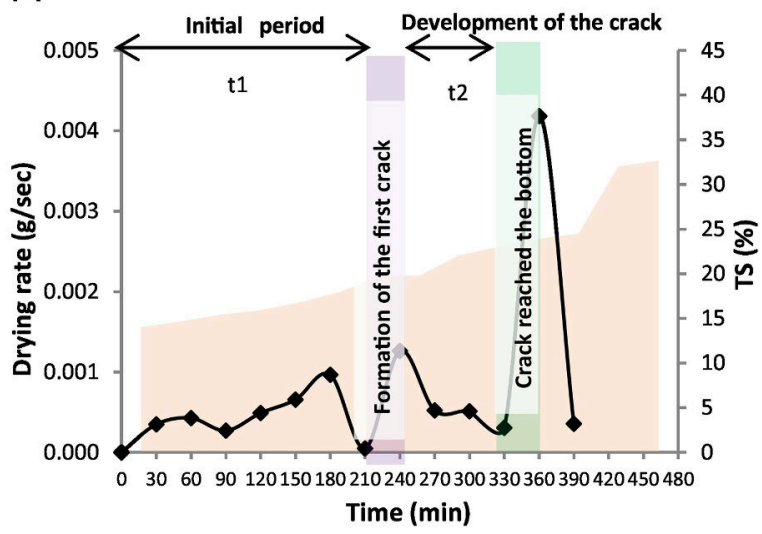

TS (\%) —Drying rate, g/sec

(d)

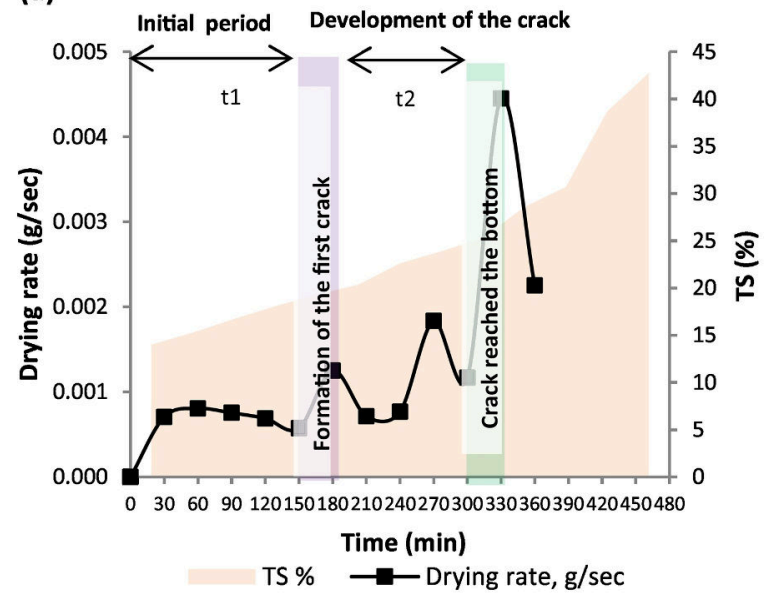

(f) 